\title{
Hereditary colorectal cancer syndromes and the COVID-19 pandemic: results from a survey conducted in patients enrolled in a dedicated registry
}

\author{
Stefano Signoroni ${ }^{1}$. Chiara Maura Ciniselli ${ }^{2}$ - Guglielmo Niccolò Piozzi ${ }^{3}$ - Maria Teresa Ricci ${ }^{1}$. Marta Pastori ${ }^{2}$. \\ Paolo Merola ${ }^{4} \cdot$ Marco Bellazzi $^{5} \cdot$ Tatiana Smaldone $^{1} \cdot$ Giuseppe Rosito $^{5} \cdot$ Claudia Borreani $^{6} \cdot$ Paolo Verderio $^{2}$ (1) . \\ Giovanni Apolone ${ }^{4} \cdot$ Marco Vitellaro ${ }^{1,3}$
}

Accepted: 9 August 2021 / Published online: 23 August 2021

(c) The Author(s), under exclusive licence to Springer Nature Switzerland AG 2021

\begin{abstract}
Purpose The coronavirus 2019 (COVID-19) pandemic has had profound consequences also for non-infected patients. This study aimed to evaluate the impact of the pandemic on the quality of life of a population with hereditary gastrointestinal cancer predisposition syndromes and on the surveillance/oncological care program of patients enrolled in a dedicated registry. Methods The study was conducted by means of an online self-report survey during the first Italian national lockdown. The survey comprised four sections: demographics; perception/knowledge of COVID-19; impact of the COVID-19 pandemic on surveillance and cancer care; health status (SF-12 questionnaire).

Results 211 complete questionnaires were considered. $25.12 \%$ of respondents reported being not at all frightened by COVID$19,63.98 \%$ felt "not at all" or "a little" more fragile than the healthy general population, and $66.82 \%$ felt the coronavirus to be no more dangerous to them than the healthy general population. $88.15 \%$ of respondents felt protected knowing they were monitored by a team of dedicated professionals.

Conclusion Patients with hereditary gastrointestinal cancer predisposition syndromes reported experiencing less fear related to COVID-19 than the healthy general population. The study results suggest that being enrolled in a dedicated registry can reassure patients, especially during health crises.
\end{abstract}

Keywords Hereditary gastrointestinal cancer syndromes $\cdot$ Familial polyposis $\cdot$ Lynch syndrome $\cdot$ Coronavirus pandemic . Registry $\cdot$ SF-12 questionnaire

\section{Introduction}

Stefano Signoroni and Chiara Maura Ciniselli equally contributed as co-first author.

Giovanni Apolone and Marco Vitellaro equally contributed as co-last author.

Paolo Verderio

paolo.verderio@istitutotumori.mi.it

1 Unit of Hereditary Digestive Tract Tumors, Fondazione IRCCS Istituto Nazionale dei Tumori, Via Venezian 1, Milan, Italy

2 Unit of Bioinformatics and Biostatistics, Fondazione IRCCS, Istituto Nazionale dei Tumori di Milano, Via Venezian 1, Milan, Italy

3 Colorectal Surgery Division, Fondazione IRCCS Istituto Nazionale dei Tumori, Via Venezian 1, Milan, Italy
International awareness of a new, rapidly spreading infectious disease caused by a novel coronavirus (COVID-19) emerged in December 2019 after an outbreak in Wuhan, China [1-4]. COVID-19 was declared a global pandemic

4 Scientific Directorate, Fondazione IRCCS Istituto Nazionale dei Tumori, Via Venezian 1, Milan, Italy

5 Information and Communication Technology Unit, Fondazione IRCCS Istituto Nazionale dei Tumori, Via Venezian 1, Milan, Italy

6 Clinical Psychology Unit, Fondazione IRCCS Istituto Nazionale dei Tumori, Via Venezian 1, Milan, Italy 
by the World Health Organization on March 11th. Italy was the first western country to adopt drastic measures to limit the spread of the virus with a full lockdown of the country. Phase 1 corresponded to a national lockdown and was in place from March 9th to May 3rd. Phase 2 was characterized by the gradual loosening of the containment measures and lasted from May 4th to June 14th. Phase 3, also known as "COVID-19 coexistence," started on June 15th. After the initial panic, the country soon adapted to the lockdown, but the impact on economic, social, and health (non-COVID-19 related) aspects of society was severe. In September 2020, the second wave of COVID-19 cases throughout Europe made new restrictions necessary. At the time of writing (November 2020), Italy is enduring a second wave with a partial national lockdown.

At the Fondazione IRCCS Istituto Nazionale dei Tumori of Milan (National Cancer Institute), which is a national cancer referral center, the Unit of Hereditary Digestive Tract Tumors (Tumori Ereditari Apparato Digerente-TEAD) manages the surveillance and treatment of patients affected by hereditary cancer predisposition syndromes, mainly Lynch syndrome and familial adenomatous polyposis (FAP) [5-9], from all regions of Italy. The unit keeps a prospectively maintained registry of patients with such syndromes.

The registry was created in the late 1980s and has since been a point of reference for patients and their families. The TEAD unit not only deals with the genetic diagnosis of the syndromes but also organizes the necessary checks for all family members and plans surgical interventions for prevention and treatment. Remote assistance, as the standard of care, is provided by phone calls and emails. Patients who undergo surveillance visits far away from Milan send the results to the registry secretariat by email; after discussion by a multidisciplinary team, which takes place once a week, they are contacted to schedule the next checks.

During Phase 1, the assistance for these patients was guaranteed with some limitations. Clinical visits, genetic counseling, and follow-up examinations were postponed if not urgent. However, the TEAD staff maintained active remote contact with patients about their needs through phone calls and emails, providing indications for them to attend clinical/ instrumental examinations close to home when necessary. Patients belonging to more than 300 families were contacted and had the opportunity to speak on the phone with doctors and staff of the TEAD unit, who reassured them by rescheduling visits that were canceled due to the pandemic and answering questions regarding their hereditary conditions and COVID-19. In more urgent cases, patients were scheduled to be examined in person after having undergone a swab test; those outside the Lombardy region were referred to centers closer to their homes.
As reported also by other authors, the rise in the levels of anxiety related to COVID-19 [10-12] was caused by the lack of adequate therapy to cope with the severe symptoms of the disease and by uncertainty about the timing of implementation of adequate vaccines [13]. Considerable effort was made to help this population cope with the anxiety following restrictions to healthcare access due to the high risk of getting infected in hospitals and to the overload of the healthcare system in northern Italy caused by COVID-19.

To our knowledge, no previous study has reported on the impact of the COVID-19 pandemic on a population with hereditary gastrointestinal cancer predisposition syndromes. These are patients who may never become ill but have an obvious need for recurrent examinations. The annual examination is a point of reference to these patients that keeps them in balance between health and disease. The aim of this study was to evaluate the impact of the pandemic, through the use of self-report standardized (SF-12) and homemade questionnaires, on the quality of life of a population with hereditary gastrointestinal cancer predisposition syndromes enrolled in a dedicated registry.

\section{Material and methods}

\section{Study description and participants}

The study was conducted through a homemade questionnaire developed by TEAD at the National Cancer Institute. The survey, approved by the Ethics Committee (INT 97/20), was managed by a multidisciplinary group including the TEAD team, institutional psychologists, biostatisticians, and computer technicians. All participants were selected from the TEAD registry. The inclusion criteria for patients were (1) having an identified pathogenic variant in a gene responsible for gastrointestinal cancer predisposition syndromes such as APC, MUTYH, POLE, SMAD4, $M L H 1, M S H 2, M S H 6, P M S 2$, or $C D H 1$ [14]; (2) providing written consent for research purposes; (3) being under active follow-up; (4) being $\geq 18$ years of age.

Data were collected anonymously online using the LimeSurvey tool (LimeSurvey GmbH, Hamburg, Germany. http://www.limesurvey.org) hosted on the institute's servers. The survey opened on May 26th and closed on June 15th after two reminders. It was conducted during Phase 2 of the national lockdown.

The invitation email contained a brief explanation of the questionnaire and instructions for filling it in as well 
as a link to the online questionnaire. Participants had to answer an "accept/don't accept" question to confirm their willingness to contribute voluntarily. After accepting, the participant was directed to complete the self-report questionnaire.

\section{Questionnaire}

The questionnaire consisted of four main sections (Supplementary Material 1): the first focused on demographics, the second on the perception/knowledge of COVID-19, and the third on the impact of the pandemic on patient surveillance and cancer care. The fourth section surveyed self-reported health status $[15,16]$.

Briefly, the first section served to obtain demographic and socioeconomic data. Data concerning age, sex, marital status, and offspring as well as education and employment were recorded together with the type of hereditary digestive disease. The second section, which is part of an ongoing larger study in collaboration with the clinical psychologists of the institute, explored participants' perception of the risks related to the coronavirus. The third section collected information about participants' knowledge of the role of a registry and a multidisciplinary team in the management of surveillance in individuals at higher risk of developing cancer. The fourth section consisted of the SF-12 standardized questionnaire, which evaluates physical (PCS12) and mental component summary (MCS12) scores through 12 questions [17].

\section{Statistics}

First, a data cleaning process was implemented to check the completeness and conformity of the data. Regarding the SF-12 questionnaire (Supplementary Material 1-Section 4), responses to each question were scored and combined to generate the two component summary scores. PCS12 and MCS12 were computed using the U.S. general population coefficients and their distribution was compared with that of a national cohort $[17,18]$. Descriptive statistics or frequency distribution tables were computed to describe the data collected in Supplementary Material 1-Sections 1, 3 and 4 according to the nature of the pivotal variable. The relationships between the two component summary scores and the impact of the COVID-19 pandemic on surveillance and cancer care (Supplementary Material 1-Section 3) were investigated by means of the non-parametric Kruskal-Wallis test. A Bonferroni correction p-value was computed to account for multiple comparisons. Specific comparisons between the two component summary scores and items from Supplementary Material 1-Section 2 were also performed. All analyses were done with the SAS software (version 9.4; SAS Institute, Inc., Cary, NC) by adopting a nominal significance level of 5\%; graphical representations were obtained with the R software.

\section{Results}

\section{Descriptive analysis}

A total of 315 invitations to participate in the survey were sent by email. At the scheduled deadline (June 15th 2020), 245 questionnaires had been received (response rate: $77.7 \%$ ). After the data cleaning process, 211 complete questionnaires were accepted and 34 were excluded: 18 did not have Supplementary Material 1-Sections 1-3 completed, while 16 had all SF-12 items missing (Supplementary Fig. 1).

Table 1 displays the clinical and demographic characteristics of the study sample $(n=211)$. Just over half of respondents were women and two-thirds belonged to the 31-60-year age group. The most frequent syndrome among respondents

Table 1 Clinical and demographic characteristics of the study sample

\begin{tabular}{|c|c|c|}
\hline & $n$ & $\%$ \\
\hline \multicolumn{3}{|l|}{ Disease recorded in the registry } \\
\hline Lynch syndrome & 126 & 59.72 \\
\hline Familial polyposis & 82 & 38.86 \\
\hline Other & 3 & 1.42 \\
\hline \multicolumn{3}{|l|}{ Sex } \\
\hline Female & 120 & 56.87 \\
\hline Male & 91 & 43.13 \\
\hline \multicolumn{3}{|l|}{ Age } \\
\hline $18-30$ & 37 & 17.54 \\
\hline $31-60$ & 143 & 67.77 \\
\hline $60+$ & 31 & 14.69 \\
\hline \multicolumn{3}{|l|}{ Children } \\
\hline Yes & 117 & 55.45 \\
\hline No & 94 & 44.55 \\
\hline \multicolumn{3}{|l|}{ Marital status } \\
\hline Single & 51 & 24.17 \\
\hline Married & 111 & 52.61 \\
\hline Cohabiting & 27 & 12.8 \\
\hline Separated & 9 & 4.27 \\
\hline Divorced & 10 & 4.74 \\
\hline Widowed & 3 & 1.42 \\
\hline \multicolumn{3}{|l|}{ Educational level } \\
\hline Elementary school & 4 & 1.9 \\
\hline Middle school & 47 & 22.27 \\
\hline High school diploma & 107 & 50.71 \\
\hline University degree & 53 & 25.12 \\
\hline \multicolumn{3}{|l|}{ Employment status } \\
\hline Retired & 25 & 11.85 \\
\hline Employed & 142 & 67.3 \\
\hline Unemployed & 21 & 9.95 \\
\hline Unemployed and looking for work & 8 & 3.79 \\
\hline Other & 15 & 7.11 \\
\hline
\end{tabular}


was Lynch syndrome, followed by FAP and a small number of other syndromes. A majority of respondents had a highschool diploma or university degree and two-thirds were employed.

As reported in Table 2, almost two-thirds of these individuals with cancer predisposition syndromes felt not at all or only a little more fragile than the healthy general population [S3.1. Because of your increased risk of cancer due to a genetic predisposition, do you generally feel more fragile than the general population?], and a similar proportion felt the coronavirus to be no more dangerous to them than the healthy general population. More than half had a followup visit scheduled in this period and almost $70 \%$ of these had already rescheduled their visits. A large majority of respondents were aware that the frequency of follow-up, regardless of where the exams were performed, was always established by a team of dedicated professionals. Moreover, most respondents felt protected in knowing they were monitored by a team of dedicated professionals who, based on the personal and family medical history of each patient, assessed whether or not to delay an exam because of the COVID-19 emergency. The knowledge of an existing registry was not associated with any other analyzed social or demographic variables in the study.

Supplementary Fig. 2 reports the bar chart corresponding to the frequency distribution for each of the 12 items of SF-12. Figure 1 depicts the overall distribution of the two component summary scores as well as those according to the main sociodemographic characteristics (sex and age). A lower standardized mental score was observed among women (mean 42.03, standard deviation (SD) 10.77) than men (mean 46.94, SD 10.05). Higher standardized physical scores were observed in the youngest group (mean 53.69, SD 7.19) compared to the 31-60-year group (mean 50.71, SD 8.49 ) and $>60$-year group (mean 48.88, SD 7.84).

Our results showed a mean mental score of 44.15 (SD 10.72), lower than that obtained by the National Institute of Statistics (ISTAT) among the Italian general population (mean 50.07, SD 9.98) but slightly higher than that observed among the same population in the subgroup of cancer patients (mean 41.64, SD 12.84) [18]. Interestingly, we observed a higher perception of physical health (mean 50.96, SD 8.27) in our study cohort compared to the ISTAT cancer population (mean 37.87, SD 11.44) [18]. Additionally, a lower standardized mental score was observed in female versus male respondents, in agreement with the data of the general ISTAT cohort, and higher standardized physical scores were observed in the youngest age group with respect to the older groups [18]. By contrast, mental scores increased with increasing age, suggesting greater awareness of one's own health status and less insecurity about the future among older individuals.

\section{Association analysis}

When we evaluated the impact of the COVID-19 pandemic on surveillance and cancer care, we found significant associations for both PCS12 and MCS12 and item S3.1. [Because of your increased risk of cancer due to a genetic predisposition, do you generally feel more fragile than the general population?] (Fig. 2A); similarly, for S3.4. [Do you think that the COVID-19 (coronavirus) epidemic is more dangerous for you than the general population?] we observed higher physical and mental scores in persons feeling "not at all" or "a little" fragile or at risk compared to those responding "very much" or "much" (Fig. 2B). This evidence is corroborated when we look at item S2.1. [Is coronavirus (COVID-19) scaring you?], with $24.64 \%$ of respondents declaring to be "not at all" or "a little" scared and $21.81 \%$ to be "much" or "very much" scared by COVID-19. Those reporting to be not at all scared by COVID-19 obtained a higher mental score value (mean 51.88 , SD 4.56$)$ than those reporting to be very scared (mean 39.20, SD 12.00) (Fig. 2C).

Looking at the medical follow-up questions, we observed significantly higher mental scores (Fig. 3A) in patients who declared being "a little" or "not at all" anxious regarding the longer time between one medical check and the next [S3.3. Generally, does lengthening the time between one medical check and the next cause you anxiety?]; likewise, in respondents who did not cancel the planned medical checks due to the pandemic (Fig. 3B), higher scores were observed for item S3.5. [During this period, did you have any planned medical checks that you could not attend due to the COVID-19 health emergency?]. Among the subgroup of 119 patients with a scheduled visit canceled because of COVID-19 [S3.5.1. Did not being able to attend the planned medical checks because of the COVID-19 emergency cause you anxiety?], the mental score was significantly higher in respondents who declared being "not at all anxious about not having done the planned checks" than in those reporting being "very anxious about not having done the planned checks" (Fig. 3C).

Finally, a higher physical score (median 54.52, range 22.39-63.66) was observed in respondents who knew about the TEAD registry [S3.6. Are you aware that within the Unit of Hereditary Digestive Tract Tumors of the IRCCS National Cancer Institute of Milan where you are under surveillanceltreatment, there is a registry dedicated to the management (surveillance and treatment) of your condition?] than in those who did not, albeit with borderline significance (raw $p$ value: 0.043 ). Supplementary Table 1 reports the main descriptive statistics for each of the comparisons. 


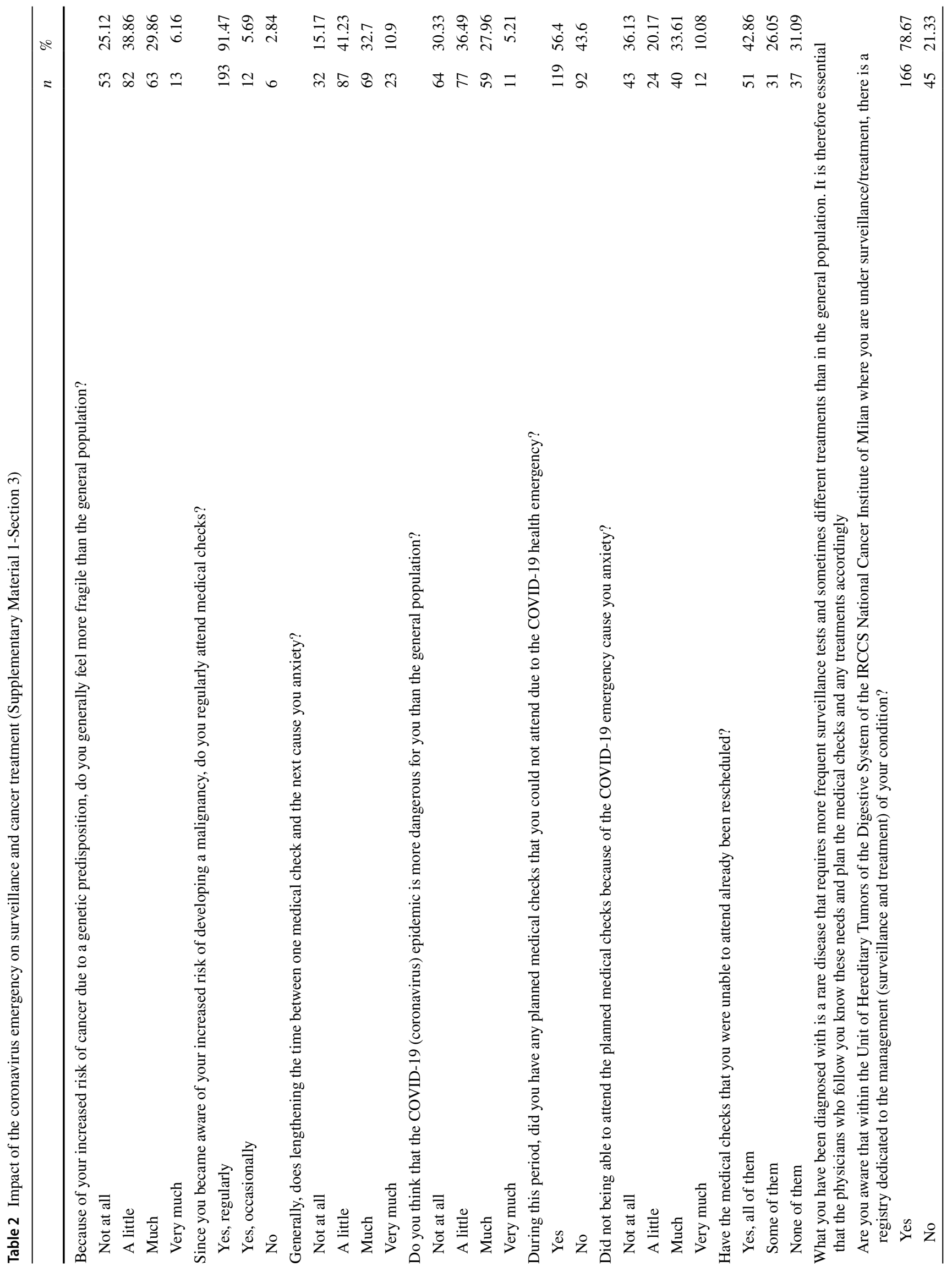




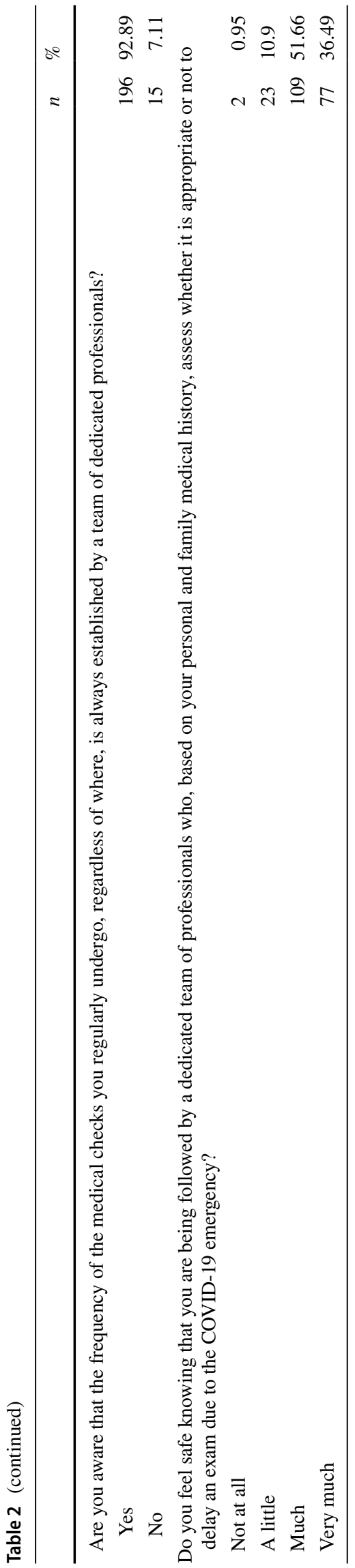

\section{Discussion}

The current pandemic has struck not only COVID-19 patients but also a large, uncounted number of patients who suffered diagnostic and treatment delays for nonCOVID-19 diseases. The limits of the local health providers have been highlighted also for cancer patients and individuals at a higher risk of cancer.

The published results of polyposis registries established between the 1970s and 1990s showed that the prognosis of FAP patients has improved [19, 20]. Other authors reported that central registration of patients combined with prophylactic examinations led to reduced mortality from colorectal cancer [21-25]. Bülow et al. reported that colorectal cancer is diagnosed in $67 \%$ of FAP probands but in only 5\% of call-up patients [26]. Reports from European registries showed that survival rates were higher in call-up patients than probands [22, 23, 27, 28].

The present study supports the usefulness of a registry for high-risk individuals, such as those with cancer predisposition syndromes, to ensure effective surveillance. For each patient under surveillance the registry provides clinicians with updated information about the risk of cancer linked to the patient's genetic predisposition. In exceptional situations such as a pandemic, it allows clinicians to ascertain, also on the basis of the last recorded visit, whether an examination needs to be performed shortly or can be postponed. Thanks to the registry, the TEAD staff was able to maintain active contact with patients from all over the country by means of phone calls or emails. The registry was useful not only for contacting patients and delivering tailored advice, but also for providing active support and reassurance by rescheduling visits when needed. Seventy-eight percent of study participants were in fact aware that there was a TEAD registry dedicated to the management of their condition, and 93\% realized that the frequency of follow-up was always established by a team of dedicated professionals. It is worthy of note that $88 \%$ felt protected knowing they were monitored by a team of professionals who, based on their personal and family history, assessed whether or not to delay an exam due to the COVID-19 emergency. Interestingly, our study shows that only $36 \%$ of patients enrolled in the registry felt more fragile with respect to COVID-19 exposure as a result of their clinical condition. Moreover, $25 \%$ of respondents reported being not at all frightened by COVID-19, a finding most likely related to the chronic stress associated with a cancer predisposition syndrome. A possible interpretation of this result, supported by what patients said during visits or contact by phone, is that the COVID-19 pandemic scares them less than cancer. According to the patients, sooner or later a vaccine against COVID-19 will 


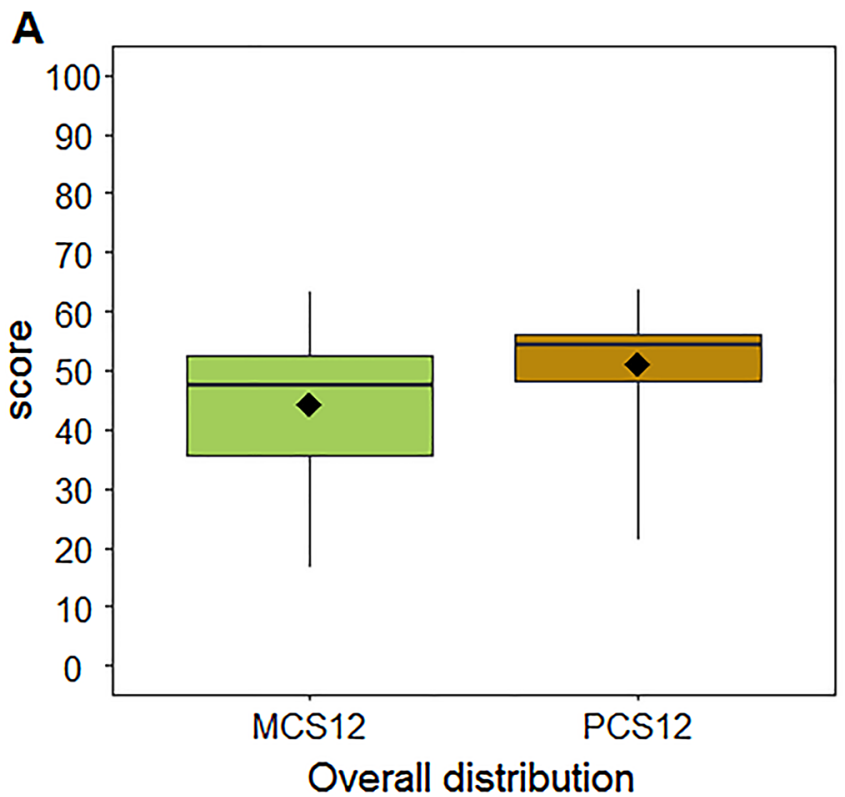

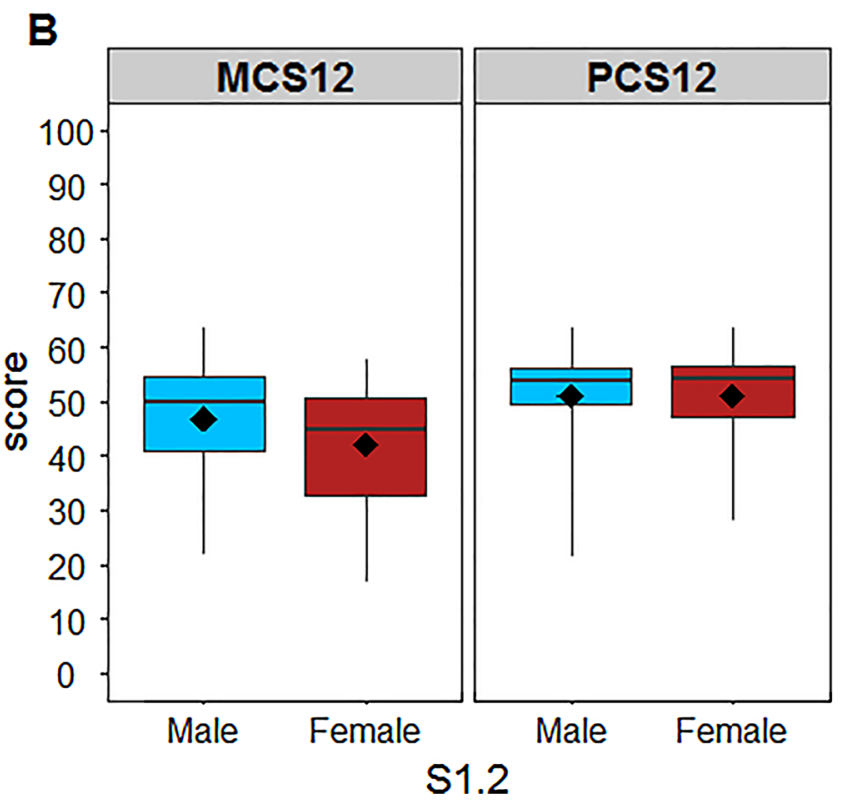

Fig. 1 Distribution of the two component summary scores (MCS12 and PCS12). The box plots report the overall distribution of the two component summary scores (A) and the distribution of the two component summary scores according to sex $(\mathbf{B})$ and age class $(\mathbf{C})$. Each

be developed (as has in fact happened), while for a long time there has been talk of a "vaccine" against cancer, which has still not materialized. Accordingly, respondents who showed no fear reported higher mental and physical scores than those who felt more fragile with respect to

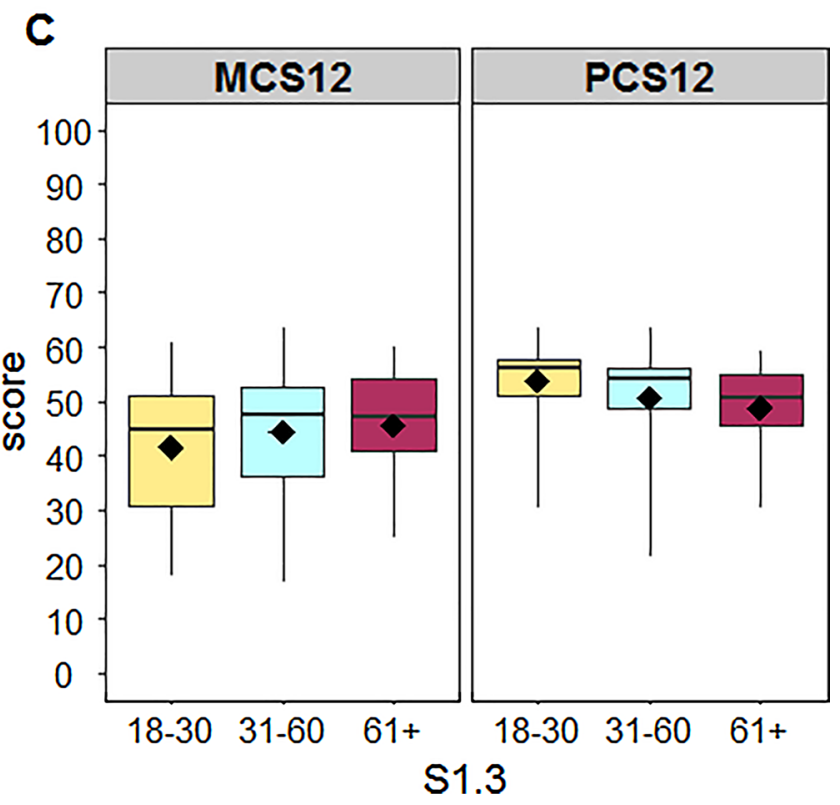

box indicates the 25 th and 75 th centiles. The horizontal line inside the box indicates the median, the black dot represents the mean, and the whiskers indicate the range measured

the healthy general population. Respondents who had to delay planned medical checks due to the pandemic showed lower mental scores, but those who were not anxious about rescheduling had much higher mental scores than those who were very anxious. Awareness of the availability of a 
A

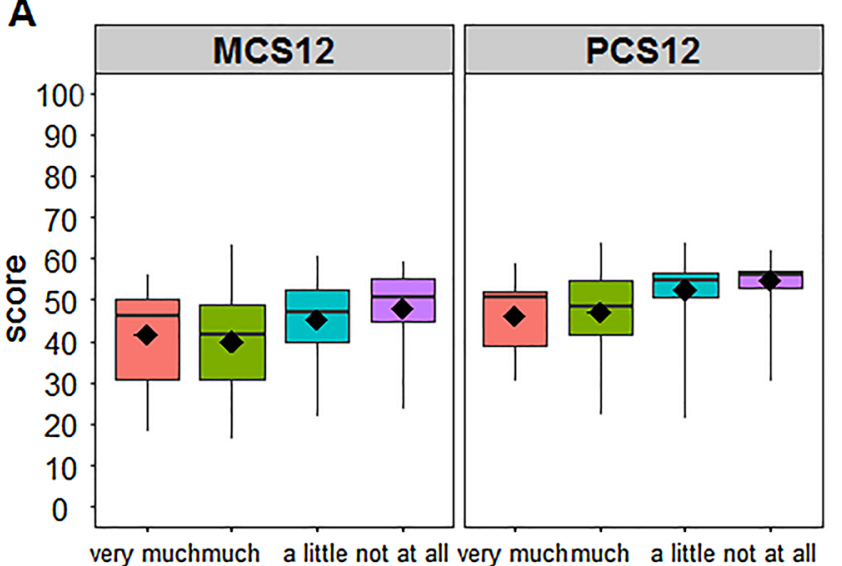

S3.1
B

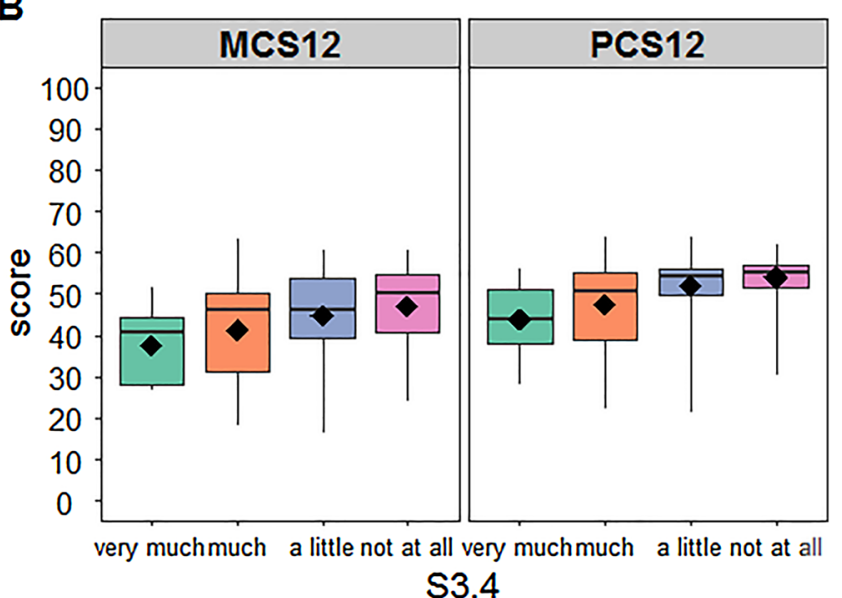

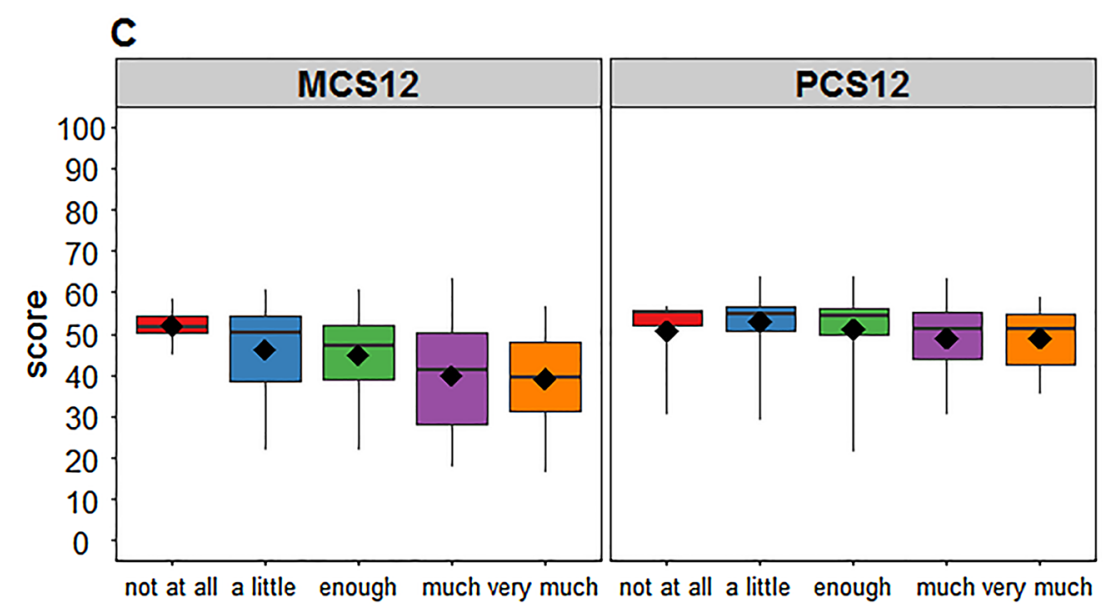

S2.1

Fig. 2 Distribution of the two component summary scores (MCS12 and PCS12) and the impact of the coronavirus emergency on surveillance and cancer treatment. The box plots report the distribution of the two component summary scores according to (A) S3.1. [Because of your increased risk of cancer due to a genetic predisposition, do you generally feel more fragile than the general population?], (B)

dedicated registry reassured the patients, leading to higher physical scores.

The rise in COVID-19 cases and the occurrence of a second wave with the implementation of a partial national lockdown introduced new difficulties in providing surveillance support to patients with hereditary conditions predisposing to cancer. However, the presence of a registry and the immediate activation of remote assistance made it possible to cope with this new health crisis. The model developed during Phase 1 of the pandemic, supported by the results of this study, proved to be effective and feasible, thanks to the presence of a well-organized registry. In September 2020, because of the COVID-19 pandemic in the Lombardy region, the "televisit" mode was introduced for
S3.4. [Do you think that the COVID-19 (coronavirus) epidemic is more dangerous for you than the general population?], and (C) S2.1. [Is coronavirus (COVID-19) scaring you?]. Each box indicates the 25 th and 75th centiles. The horizontal line inside the box indicates the median, the black dot represents the mean, and the whiskers indicate the range measured

patients under surveillance and the TEAD unit immediately adopted this modality.

A limitation of our study is that, due to the survey's anonymity, it was not possible to investigate the characteristics of non-responders. In addition, the electronic format could be an obstacle for those not familiar with computers and the internet, such as elderly patients. Furthermore, patients were not asked how long they had been in the registry. This could be an intervening variable between the awareness of belonging to a registry and certain outcomes of the studyfor example, the physical scores, which showed a borderline level of significance. In fact, it is conceivable that people who had been in the registry longest had grown more confident about its benefits for their management. 


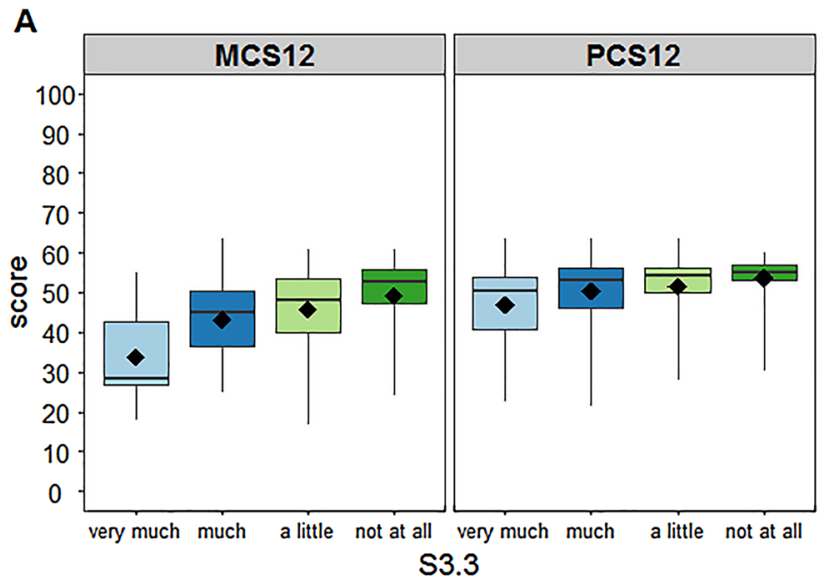

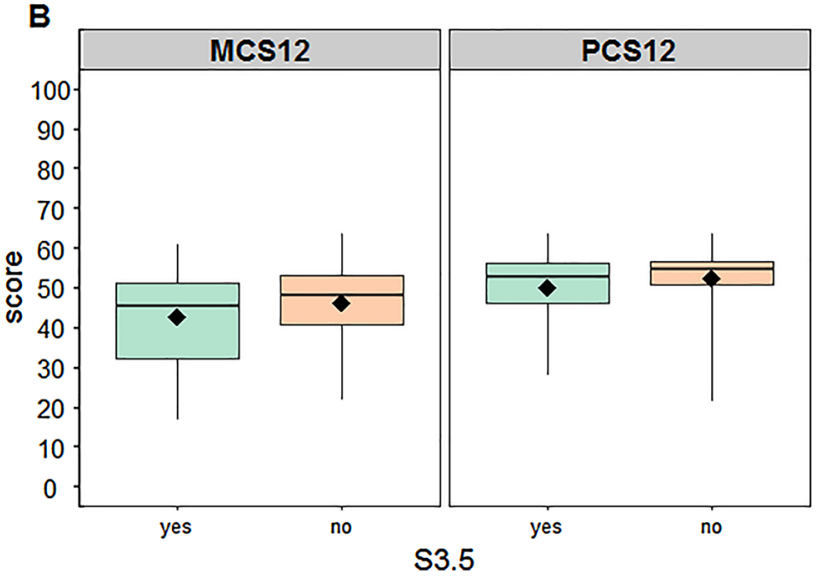

Fig. 3 Distribution of the two component summary scores (MCS12 and PCS12) and the impact of the coronavirus emergency on medical follow-up. The box plots report the distribution of the two component summary scores according to (A) S3.3. [Generally, does lengthening the time between one medical check and the next cause you anxiety?], (B) S3.5. [During this period, did you have any planned medical

In conclusion, this study showed that patients with hereditary gastrointestinal cancer predisposition syndromes reported experiencing less fear related to COVID-19 than the healthy general population. The study also suggests that these patients, when included in a regular surveillance program (registry), feel confident in knowing that their surveillance is programmed by a team of experts that follows them, even at a distance and during a pandemic crisis.

Supplementary Information The online version contains supplementary material available at https://doi.org/10.1007/s11136-021-02973-4.

Acknowledgements The authors wish to acknowledge Mariangela Di Ceglie and Ornella Galuppo of the Hereditary Digestive Tract Tumors Unit for prospectively maintaining the patient database. Special thanks go to Dr. Sara Alfieri of the Clinical Psychology Unit at Fondazione IRCCS Istituto Nazionale dei Tumori for her help and suggestions in revising the text. The authors also express sincere thanks to the Italian patients and families of the Association of Patients with Hereditary Digestive Tract Tumors-APTEAD—for their support in the

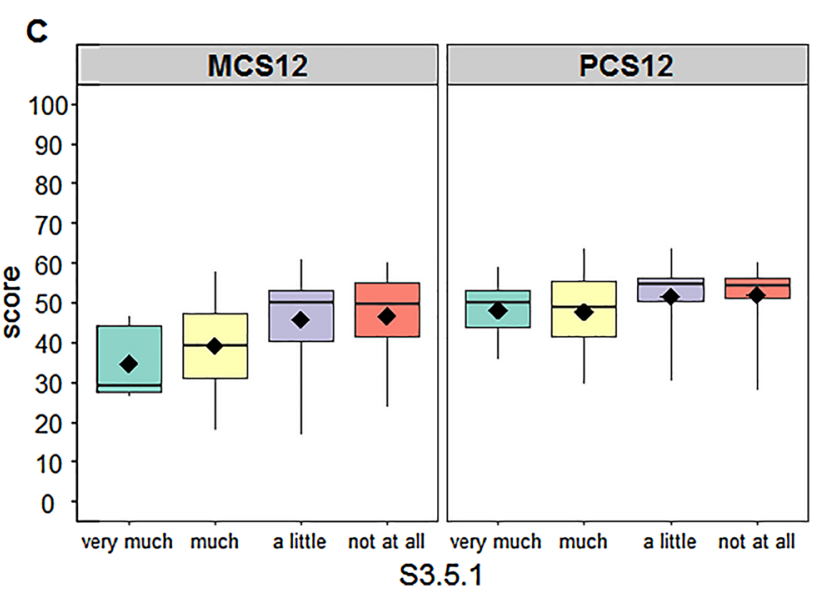

checks that you could not attend due to the COVID-19 health emergency?], and (C) S3.5.1. [Did not being able to attend the planned medical checks because of the COVID-19 emergency cause you anxiety?]. Each box indicates the 25th and 75th centiles. The horizontal line inside the box indicates the median, the black dot represents the mean, and the whiskers indicate the range measured

distribution of the questionnaire. Marije de Jager is acknowledged for editing assistance.

Funding This research did not receive any specific grant from funding agencies in the public, commercial, or not-for-profit sectors.

\section{Declarations}

Conflict of interest The authors have no conflicts of interest to declare.

Ethical approval The study was approved by the Ethics Committee of Fondazione IRCCS Istituto Nazionale dei Tumori di Milano (INT 97/2020)

Consent to participate Participants expressed their willingness to contribute voluntarily. Data were collected anonymously online using the LimeSurvey tool (LimeSurvey GmbH, Hamburg, Germany. http:// www.limesurvey.org). 


\section{References}

1. Deng, X., Yang, J., Wang, W., Wang, X., Zhou, J., Chen, Z., Li, J., Chen, Y., Yan, H., Zhang, J., Zhang, Y., Wang, Y., Qiu, Q., Gong, H., Wei, X., Wang, L., Sun, K., Wu, P., Ajelli, M., ... Yu, H. (2020). Case fatality risk of the first pandemic wave of novel coronavirus disease 2019 (COVID-19) in China. Clinical Infectious Diseases, 73(1), e79-e85.

2. Chen, N., Zhou, M., Dong, X., Qu, J., Gong, F., Han, Y., Qiu, Y., Wang, J., Liu, Y., Wei, Y., Xia, J., Yu, T., Zhang, X., \& Zhang, L. (2020). Epidemiological and clinical characteristics of 99 cases of 2019 novel coronavirus pneumonia in Wuhan, China: A descriptive study. Lancet, 395(10223), 507-513.

3. Wang, C., Horby, P. W., Hayden, F. G., \& Gao, G. F. (2020). A novel coronavirus outbreak of global health concern. Lancet, 395(10223), 470-473.

4. Li, Q., Guan, X., Wu, P., Wang, X., Zhou, L., Tong, Y., Ren, R., Leung, K. S. M., Lau, E. H. Y., Wong, J. Y., Xing, X., Xiang, N., Wu, Y., Li, C., Chen, Q., Li, D., Liu, T., Zhao, J., Liu, M., \& Feng, Z. (2020). Early transmission dynamics in Wuhan, China, of novel coronavirus-infected pneumonia. New England Journal of Medicine, 382(13), 1199-1207.

5. Vasen, H. F., Moslein, G., Alonso, A., Aretz, S., Bernstein, I., Bertario, L., Blanco, I., Bülow, S., Burn, J., Capella, G., Colas, C., Engel, C., Frayling, I., Friedl, W., Hes, F. J., Hodgson, S., Jarvinen, H., Mecklin, J. P., Moller, P., \& Wijnen, J. (2008). Guidelines for the clinical management of familial adenomatous polyposis (FAP). Gut, 57(5), 704-713.

6. Gupta, S., Provenzale, D., Llor, X., Halverson, A. L., Grady, W., Chung, D. C., Haraldsdottir, S., Markowitz, A. J., Slavin, T. P., Jr., Hampel, H., CGC, Ness, R. M., Weiss, J. M., Ahnen, D. J., Chen, L. M., Cooper, G., Early, D. S., Giardiello, F. M., Hall, M. J., ... Ogba, N. (2019). NCCN guidelines insights: Genetic/familial high-risk assessment: Colorectal, version 2.2019. Journal of the National Comprehensive Cancer Network, 17(9), 1032-1041.

7. Friedl, W., \& Vogt, S. (Eds.). (2009). Gastrointestinal Polyposis Syndromes: Hereditary Tumors, From Genes to Clinical Consequences. Wiley.

8. Möslein, G. (2009). Lynch Syndrome (HNPCC). Hereditary Tumors From Genes to Clinical Consequences (pp. 281-294). Weinheim: Wiley.

9. Garre, P., Martin, L., Bando, I., Tosar, A., Llovet, P., Sanz, J., Romero, A., de la Hoya, M., Diaz-Rubio, E., \& Caldes, T. (2013). Cancer risk and overall survival in mismatch repair proficient hereditary non-polyposis colorectal cancer, Lynch syndrome and sporadic colorectal cancer. Familial Cancer, 13(1), 109-119.

10. Molica, M., Mazzone, C., Cordone, I., Pasquale, A., Niscola, P., $\&$ de Fabritiis, P. (2020). SARS-CoV-2 infection anxieties and general population restrictions delay diagnosis and treatment of acute haematological malignancies. British Journal of Haematology, 190(1), e5-e8.

11. Mazza, C., Ricci, E., Biondi, S., Colasanti, M., Ferracuti, S., Napoli, C., \& Roma, P. (2020). A nationwide survey of psychological distress among Italian People during the COVID-19 pandemic: Immediate psychological responses and associated factors. International Journal of Environmental Research and Public Health, 17(9), 3165.

12. Wang, C., Pan, R., Wan, X., Tan, Y., Xu, L., Ho, C. S., \& Ho, R. C. (2020). Immediate psychological responses and associated factors during the initial stage of the 2019 coronavirus disease (COVID-19) epidemic among the general population in China. International Journal of Environmental Research and Public Health, 17(5), 1729.
13. Fauci, A. S., Lane, H. C., \& Redfield, R. R. (2020). Covid-19Navigating the Uncharted. New England Journal of Medicine, 382(13), 1268-1269.

14. Ricci, M. T., Volorio, S., Signoroni, S., Mariani, P., Mariette, F., Sardella, D., Pensotti, V., \& Vitellaro, M. (2019). Development, technical validation, and clinical application of a multigene panel for hereditary gastrointestinal cancer and polyposis. Tumori, 105(4), 338-352.

15. Gandek, B., Ware, J. E., Aaronson, N. K., Apolone, G., Bjorner, J. B., Brazier, J. E., Bullinger, M., Kaasa, S., Leplege, A., Prieto, L., \& Sullivan, M. (1998). Cross-validation of item selection and scoring for the SF-12 Health Survey in nine countries: results from the IQOLA Project. International quality of life assessment. Journal of Clinical Epidemiology, 51(11), 1171-1178.

16 Kodraliu, G., Mosconi, P., Groth, N., Carmosino, G., Perilli, A., Gianicolo, E. A., Rossi, C., \& Apolone, G. (2001). Subjective health status assessment: evaluation of the Italian version of the SF-12 Health Survey. Results from the MiOS Project. Journal of Epidemiology and Biostatistics, 6(3), 305-316.

17. Ware, J., Jr., Kosinski, M., \& Keller, S. D. (1996). A 12-item short-form health survey: Construction of scales and preliminary tests of reliability and validity. Medical Care, 34(3), 220-233.

18. Apolone, G., Mosconi, P., Quattrociocchi, L., Gianicolo, E. A. L., Groth, N., \& Jr, J. E. W. (2001). Questionario sullo stato di Salute SF-12. Versione Italiana Milano: Guerini e Associati Editore.

19. Jarvinen, H. J., Husa, A., Aukee, S., Laitinen, S., Matikainen, M., \& Havia, T. (1984). Finnish registry for familial adenomatosis coli. Scandinavian Journal of Gastroenterology, 19(7), 941-946.

20 Vasen, H. F., Griffioen, G., Offerhaus, G. J., Den HartogJager, F. C., Van Leeuwen-Cornelisse, I. S., Meera Khan, P., Lamers, C. B., \& Van Slooten, E. A. (1990). The value of screening and central registration of families with familial adenomatous polyposis. A study of 82 families in The Netherlands. Diseases of the Colon \& Rectum, 33(3), 227-230.

21. Bertario, L., Presciuttini, S., Sala, P., Rossetti, C., \& Pietroiusti, M. (1994). Causes of death and postsurgical survival in familial adenomatous polyposis: results from the Italian Registry. Italian Registry of Familial Polyposis Writing Committee. Seminars in Surgical Oncology, 10(3), 225-234.

22 Bülow, S., Bülow, C., Nielsen, T. F., Karlsen, L., \& Moesgaard, F. (1995). Centralized registration, prophylactic examination, and treatment results in improved prognosis in familial adenomatous polyposis. Results from the Danish Polyposis Register. Scandinavian Journal of Gastroenterology, 30(10), 989-993.

23. Heiskanen, I., Luostarinen, T., \& Jarvinen, H. J. (2000). Impact of screening examinations on survival in familial adenomatous polyposis. Scandinavian Journal of Gastroenterology, 35(12), 1284-1287.

24. Ardoino, I., Signoroni, S., Malvicini, E., Ricci, M. T., Biganzoli, E. M., Bertario, L., Occhionorelli, S., \& Vitellaro, M. (2020). Long-term survival between total colectomy versus proctocolectomy in patients with FAP: A registry-based, observational cohort study. Tumori, 106(2), 139-148.

25. Signoroni, S., Piozzi, G. N., Ricci, M. T., Mancini, A., Morabito, A., Bertario, L., \& Vitellaro, M. (2020). Risk factors for metachronous colorectal cancer in Lynch syndrome patients: A registrybased observational mono-institutional study cohort. International Journal of Clinical Oncology, 25(9), 1644-1652.

26. Bülow, S. (2003). Results of national registration of familial adenomatous polyposis. Gut, 52(5), 742-746.

27. Gibbons, D. C., Sinha, A., Phillips, R. K., \& Clark, S. K. (2011). Colorectal cancer: No longer the issue in familial adenomatous polyposis? Familial Cancer, 10(1), 11-20. 
28. Piozzi, G. N., Ricci, M. T., Signoroni, S., \& Vitellaro, M. (2019). Ready, get set, go: Fast preoperative genetic diagnosis is the present future in Lynch syndrome surgical strategy. Digestive and Liver Disease, 51(12), 1742-1743.
Publisher's Note Springer Nature remains neutral with regard to jurisdictional claims in published maps and institutional affiliations. 